\title{
Fortalecimiento del proceso de certificación aeromédica en la Fuerza Aérea Colombiana
}

| Fecha de recibido: 27 de febrero del 2020 | Fecha de aprobación: 29 de abril del 2020 |

\author{
Luis Alberto Saavedra Martínez \\ Administrador Aeronáutico \\ Piloto comercial, Estudiante de Maestría \\ en Seguridad Operacional \\ Rol de investigador: intelectual \\ https://orcid.org/0000-0002-6071-7906 \\ $\bowtie$ Pilcomt27@hotmail.com
}

Cómo citar este artículo: Saavedra Martínez, L. (2020). Fortalecimiento del proceso de certificación aeromédica en la Fuerza Aérea Colombiana. Revista Ciencia y Poder Aéreo, 15(1), 16-23. https://doi.org/10.18667/cienciaypoderaereo.655 


\section{Fortalecimiento del proceso de certificación aeromédica en la Fuerza Aérea Colombiana}

Resumen: La certificación aeromédica en el mundo tiene un propósito primario: garantizar que el entorno aeronáutico cuente con personal capaz de operar en él de una forma segura. Los requerimientos de estados físico y mental pueden ser altos, dependiendo del ambiente en el cual se realicen las operaciones. Certificarlos contribuye sustancialmente a garantizar la seguridad, pero se requiere una revisión exhaustiva del balance, por una parte, los requisitos y procesos de certificación aeromédica y, por otra, la realidad y necesidades operativas del entorno para garantizar que los procesos sean una herramienta efectiva y no únicamente requisitos. En este artículo se plantean unas alternativas para aumentar la objetividad de las decisiones en términos de certificación. Se toma como punto de partida la propuesta de estratificar los certificados dependiendo del equipo del que se trate y de la responsabilidad en la aeronave. Se considera igualmente el fortalecimiento de la toma de decisiones aeromédicas (ADM, Aeromedical Decision Making) mediante el algoritmo propuesto por Navathe, Drane y Preitner para la Oficina de Medicina de Aviación de la Autoridad de Seguridad de la Aviación Civil en Canberra, Australia.

Palabras clave: Certificación aeromédica; seguridad operacional; toma de decisiones aeromédicas.

Abstract: Worldwide, aeromedical certification has a primary purpose: to ensure personnel capable of operating safely in the aeronautical environment. As a matter of fact, the requirements of physical and mental states may be high depending on the environment in which operations are executed. Enforcing these requisites contributes substantially to safety, although a thorough review of the balance between the requirements and processes of aeromedical certification versus the reality and operational needs of the environment is required in order to secure this becomes an effective tool and not just a list of requirements. Some alternatives are proposed to increase the objectivity of decisions in terms of certification, taking as a starting point a stratification of the certificates depending on the aircraft being operated and the position inside the cabin, as well as the strengthening of the aeromedical decision-making process (ADM) based on the algorithm proposed by Navathe, Drane, and Preitner for the Office of Aviation Medicine of the Civil Aviation Security Authority in Canberra, Australia.

Keywords: Aeromedical Certification; Aeromedical Decision Making; Operational Safety.

Resumo: No mundo, a certificação aeromédica tem um objetivo principal: garantir que o ambiente aeronáutico tenha o pessoal capaz de operar nele de uma forma segura. Os requisitos de estados físico e mental podem ser altos, dependendo do ambiente em que as operações sejam realizadas. Assegurá-los contribui substancialmente para garantir a segurança, mas requer uma revisão meticulosa do equilíbrio entre, por um lado, os requisitos e processos de certificação aeromédica e, por outro lado, a realidade e as necessidades operacionais do ambiente para assegurar que os processos sejam uma ferramenta eficiente e não apenas requisitos. Neste artigo são apresentadas alternativas para reforçar a objetividade das decisões em termos de certificação. A proposta da estratificação é considerada ponto de partida, dependendo do equipamento em questão e da responsabilidade da aeronave. Do mesmo modo, é considerado o fortalecimento da tomada de decisão aeromédica (ADM, Aeromedical Decision Making) por meio do algoritmo proposto por Navathe, Drane e Preitner para o escritório de Medicina da Autoridade de Aviação de Segurança da Aviação Civil em Canberra, Austrália.

Palavras-chave: Certificação aeromédica; Segurança operacional; Tomar decisões aeromédicas. 


\section{Introducción}

En Colombia, los procedimientos establecidos para la obtención de la certificación aeromédica para la aviación comercial, regulada por la Aerocivil (RAC 67, 2017), y los de la aviación de Estado (Fuerza Aérea Colombiana, 2016) son similares. Al realizar una comparación se encuentra que, salvo en algunas excepciones, estos procedimientos pretenden estar en concordancia y alineación con la legislación nacional y la normatividad internacional.

Se aprecia además, que la realización de exámenes y pruebas es extensa, por ejemplo, para certificar la aptitud psicofísica de un tripulante que ya ha pasado por un proceso de selección inicial de ingreso al servicio activo, la Fuerza Aérea Colombiana (FAC) realiza veintitrés exámenes. Así mismo, son necesarios otros veintiuno para la evaluación psicofísica pre-vuelo. Finalmente, se requieren entre veinte y veintiocho más (dependiendo del sexo, edad o condiciones preexistentes) para los controles periódicos (Fuerza Aérea Colombiana, 2016).

La evidencia científica (Ricaurte et al., 2016) concluye que el riesgo de accidentes o incidentes aéreos en la población de pilotos certificados es menor. Se presenta una mayor tasa de incidentes en pilotos no certificados (aviación deportiva), por lo que no se cuestiona la necesidad de realizar el proceso, menos en un medio complejo como el de la operación militar. No obstante, mediante un sustento teórico se plantea que es pertinente revisar qué tan necesarios son cada uno de los exámenes requeridos por una certificación de la FAC 0 , si se quiere, la restructuración de un proceso que ha tenido pocos cambios estructurales en el tiempo.

En una revisión de los documentos rectores (Ministerio de Defensa Nacional, 2014; Fuerza Aérea Colombiana, 2011), se encuentra que la normativa establecida hace parte de la gestión de la seguridad operacional, como lo deja ver de manera implícita el Manual de Gestión en Seguridad Operacional para la FAC. Explícitamente, el objetivo es reducir la posibilidad de que alguna condición patológica afecte la seguridad de vuelo. Sin embargo, no hay un contexto científico-investigativo que demuestre la necesidad de realizar cada uno de estos test para mantener o reducir el nivel de riesgo de accidentes causados por incapacitación fisiológica y mantenerlo en unos márgenes aceptables.

El establecimiento de un método para la realización de una investigación que dé una base teórica para el fortalecimiento del proceso de certificación aeromédica en la FAC, hace parte de un proceso que involucra varias etapas complementarias entre sí. En el presente artículo se plantean tres fases para abordar esa futura investigación, que podría ser el sustento teórico necesario para la revisión y estructuración del proceso de certificación. Dichas fases son fruto de una revisión teórica, la cual condujo a su planteamiento.

\section{Desarrollo de un sistema de información aeromédico}

Poseer un sistema de información aeromédico tiene muchas ventajas para la seguridad: a partir de este se pueden realizar estudios epidemiológicos; sirve como fuente para analizar las decisiones sobre certificación; es un insumo para realizar las matrices de riesgo de acuerdo con las estadísticas de enfermedades en una población, y para realizar análisis predictivos, entre muchas otras.

Se debe tener en cuenta que, incluso si se tienen las bases de datos, su integración no es fácil. Se requiere invertir tiempo y recursos para lograr la incorporación de los datos, que incluyen desde hojas de vida de los pacientes, hasta eventos de seguridad ocurridos; en algunos casos, se requiere incluso la realización de minería de datos. El primer paso en ese proceso es la conformación de un equipo interdisciplinario que establezca una fecha de inicio de recolección de la información a partir de un hito operacional, científico o histórico (por ejemplo, la entrada en vigencia del decreto 1796). Después de esto, se debe iniciar la recopilación de datos suficientes $\mathrm{y}$, finalmente, se debe diseñar una métrica para medir patrones patológicos en una población determinada. El trabajo realizado 
por el equipo de investigación en bioinformática del Instituto Civil de Medicina Aeroespacial (CAMI) es una muestra de este proceso. Este equipo reunió datos (de años tan lejanos como 1962) de toda la población de pilotos de Estados Unidos, recopilados de diferentes fuentes (Peterman et al., 2016).

Las ventajas del análisis de los datos va mucho más allá. Por medio del estudio de patologías predominantes en una población, es probable saber el impacto que un posible evento médico tendrá en términos de seguridad. Así se puede determinar qué exámenes son importantes y cuáles pueden ser eliminados en un proceso de recertificación. Como ejemplo, está el estudio Aeromedical Certification of Aircrew and Controllers with Renal Calculi (Drane et al., 2013), en el cual se establece una estratificación del riesgo a partir de la aplicación de un algoritmo que tiene en cuenta dentro de sus variables el tipo de vuelo (solo, cabina múltiple), para facilitar el proceso de toma de decisiones con respecto a la certificación.

\section{Evaluación del riesgo aeromédico en la FAC como medio para restructurar la certificación aeromédica}

Adoptando el método desarrollado en el estudio Assessing aeromedical risk: a three-dimensional risk matrix approach (Gray et al., 2019), se puede realizar una restructuración de los tipos de certificados y exámenes necesarios para su expedición. En dicha investigación se propone la generación de matrices de riesgo aeromédicas específicas para la operación por categorías de aeronaves (combate, transporte, helicópteros e inteligencia). Las matrices incluyen probabilidad e impacto y añaden el cargo desempeñado en la cabina, como una tercera dimensión (Gray et al., 2019).

No obstante, la evaluación de estas matrices supone un amplio conocimiento de las áreas de medicina aeroespacial, operaciones aéreas y seguridad operacional. Estas tareas son usualmente el trabajo de un equipo multidisciplinario.

Esta evaluación debe tener un fundamento científico, como en el caso de la investigación que dio origen al cálculo de la probabilidad de accidentes derivados de una incapacitación. Esta investigación fue desarrollada en el Reino Unido, y nace de la regla de un $1 \%$ de riesgo aceptable de accidentes por incapacitación en un año por cada $10^{7}$ horas de vuelo. La investigación encontró que en realidad este valor puede estar alrededor de $0,1 \%$ por cada $10^{7}$ horas de vuelo por año. Además, encontró que adicionar un piloto a la ecuación reduce el riesgo por un factor de 1000. En una cabina de dos pilotos, el riesgo de sufrir un accidente por incapacitación médica es 1 por $10^{10}$ horas por año - se contempla aquí la incapacidad por un evento médico, como un infarto, en una de las fases críticas del vuelo: despegue, ascenso, aproximación o aterrizaje; (Evans, 2016)-.

Un ejemplo simple de esta evaluación se puede hacer tomando una cabina de tripulación múltiple, como las cabinas de aeronaves de transporte de la FAC.

En una matriz de probabilidad e impacto de $4 \times 4$, en la que el impacto está definido por cuatro clases de eventos médicos ( 1 es el de mínimo impacto en el performance y 4 es la incapacitación total, véase en la tabla 1) y la probabilidad es de cuatro niveles (que van desde muy probable hasta altamente improbable), se atribuyeron valores lógicos para observar el comportamiento de la tercera variable: tripulación (representada en tres categorías diferentes: piloto-copiloto, navegante y otro cargo que no está ligado al control directo de la aeronave, como el de piloto supervisor de vuelo, maestro de carga o técnico de vuelo). Se encontró así una similitud con la realidad operacional de este tipo de equipos.

Una tercera dimensión marca la pauta para ofrecer diferentes tipos de certificados dependiendo de la influencia de cada cargo específico en la severidad del impacto dentro de una aeronave. En términos prácticos, después de un análisis por parte de la Dirección de Medicina Aeroespacial (DIMAE), un tripulante sin una función primordial en las fases críticas del vuelo 
podría recibir un certificado con menos requerimientos para su operación durante la permanencia en el cargo. La evaluación de su condición física, más que obedecer a exámenes clasificatorios, obedecería a la aplicación de un algoritmo sencillo que facilite la toma de decisiones aeromédicas, lo que eliminaría la subjetividad del ejercicio.

Tabla 1

Matriz 4x4 por cargo en una aeronave de transporte

\begin{tabular}{l|c|c|c|c|}
\hline Piloto/Copiloto & Clase 1 & Clase 2 & Clase 3 & Clase 4 \\
\hline Muy probable & 22 & 55 & 88 & 110 \\
\hline Probable & 14 & 35 & 56 & 70 \\
\hline Improbable & 6 & 15 & 24 & 30 \\
\hline Altamente improbable & 2 & 5 & 8 & 10 \\
\hline
\end{tabular}

\begin{tabular}{|l|c|c|c|c|}
\hline Navegante & Clase 1 & Clase 2 & Clase 3 & Clase 4 \\
\hline Muy probable & 22 & 30 & 35 & 70 \\
\hline Probable & 14 & 30 & 30 & 35 \\
\hline Improbable & 6 & 15 & 24 & 30 \\
\hline Altamente improbable & 2 & 5 & 8 & 10 \\
\hline
\end{tabular}

\begin{tabular}{l|c|c|c|c|}
\hline Otro cargo & Clase 1 & Clase 2 & Clase 3 & Clase 4 \\
\hline Muy probable & 22 & 30 & 30 & 35 \\
\hline Probable & 14 & 24 & 30 & 30 \\
\hline Improbable & 6 & 15 & 24 & 30 \\
\hline Altamente improbable & 2 & 5 & 8 & 10 \\
\hline
\end{tabular}

Fuente: elaboración propia.

En la actualidad, hay tres categorías en el reglamento de aptitud psicofísica de ingreso y especial para la Fuerza Aérea Colombiana (I, II y III) para el personal que ingresa a la actividad de vuelo, realiza control periódico o cambia de especialidad, o el que realiza actividades denominadas "complementarias al vuelo". Esta caracterización es susceptible de mejora si se involucran los resultados de la aplicación de matrices como la anteriormente descrita. Así, se daría lugar a una nueva tipificación que minimice el número de exámenes realizados a los aspirantes a cada tipo de certificación. A le vez que, ampliaría el espectro de personal seleccionable sin afectar la seguridad operacional.

Es necesario comparar el proceso llevado a cabo en la FAC con algunos realizados en otras entidades, que han demostrado ser igualmente efectivos. Uno de estos es el que realiza la Fuerza Aérea de los Estados Unidos (USAF), que sigue los lineamientos del Departamento de Defensa (DOD). En este proceso se establecen tipologías de pruebas basadas en la actividad que el aspirante realizará o en las características de las aeronaves. Se crean así ocho tipos de certificación. Las certificaciones exigen mayores requisitos en la medida en que el riesgo de accidentalidad por incapacitación de la tripulación sea mayor.

La USAF realiza un total de doce exámenes para el ingreso al servicio activo (United States Department of Defense, 2018; Department of the Air Force, 2013). Los exámenes van desde medicina general hasta especialidades como oftalmología, optometría, audiometría y laboratorios. Este es un número bastante reducido si se lo compara con el número de exámenes realizados en la FAC. El protocolo de la USAF es solo un ejemplo de cómo se puede hacer un proceso de ingreso más acorde a las labores que se desempeñarán.

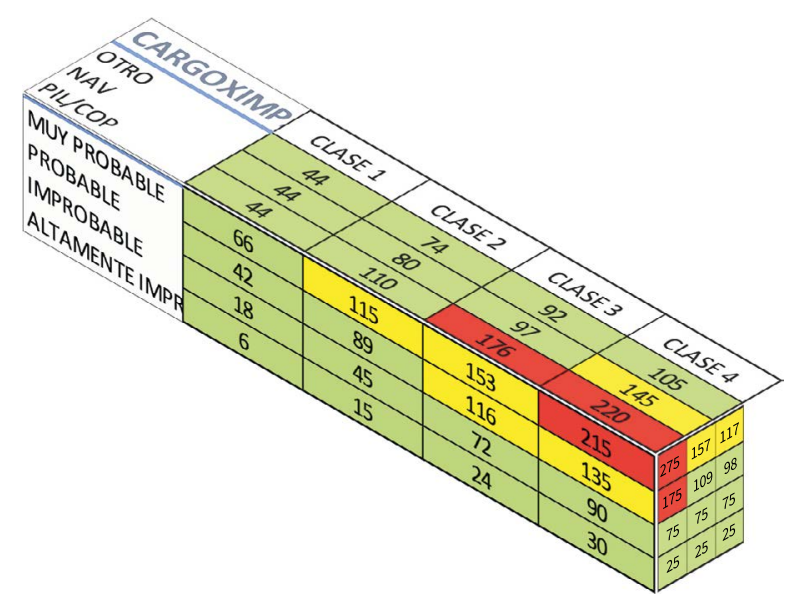

Figura 1. Representación tridimensional de las relaciones Cargo vs. Impacto, Clase vs. Probabilidad y Cargo vs. Probabilidad Fuente: elaboración propia. 


\section{Adopción o generación de algoritmos como soporte para la toma de decisiones aeromédicas}

La toma de decisiones aeromédicas se aplica en varios escenarios, como evacuaciones aeromédicas, tratamientos en teatros operacionales, certificación de tripulantes, entre otros. La certificación, a pesar de estar reglamentada y cumplir con unos parámetros definidos, siempre va a tener un componente de subjetividad, e incluso un componente emocional. Además, hay un margen de error natural en ella por tratarse de un proceso de medición.

Para minimizar los errores en la medición, existen varios desarrollos de algoritmos mundialmente conocidos que han fortalecido la ADM. Prácticamente todos ellos se fundamentan en el principio del manejo de riesgo aceptable. Si la condición psicofísica implica un riesgo mayor al nivel de riesgo aceptable entonces se busca un tratamiento antes de emitir el certificado (figura 2).

Lo que se quiere valorar con la propuesta aquí presentada (y no en la clasificación actual) es la capacidad funcional del individuo y su comparación con el tipo de certificado requerido. Un punto de partida para lograr esto, puede ser la adaptación del algoritmo de Navathe, Drane y Preitner a las necesidades institucionales. Este algoritmo contiene cinco entradas para la toma de decisiones, como se puede observar en la figura 2:

1. Determinación de la probabilidad de un evento clínicamente significativo para la condición de salud.

2. Determinación de la probabilidad de un evento de aviación indeseable derivado de la condición psicofísica.

3. Determinación de la aceptabilidad de los riesgos combinados (1 y 2 ).

4. Determinación del nivel de riesgo para la condición psicofísica después de la intervención clínica.

5. Gestión y determinación del nivel de riesgo después de las restricciones operativas para la condición psicofísica.

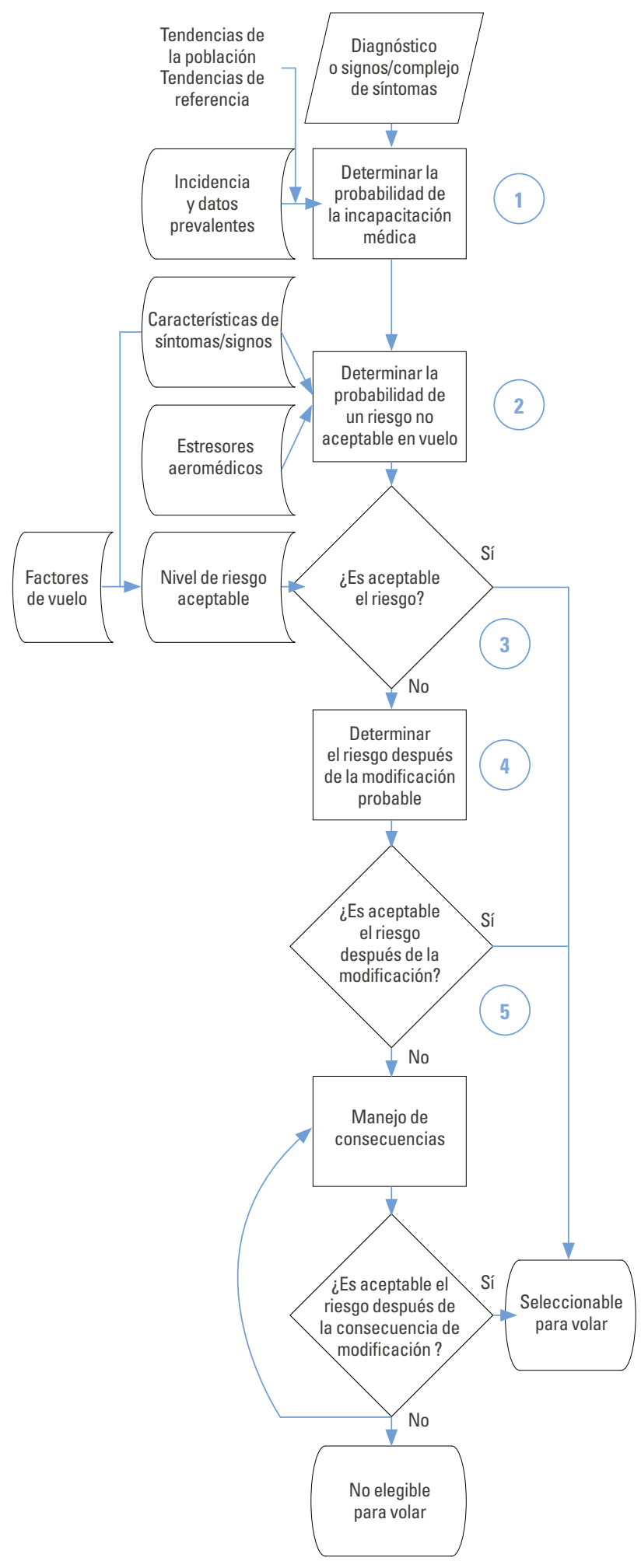

Figura 2. Algoritmo de Navathe, Drane y Preitner. Regulación de toma de decisiones aeromédicas Fuente: Navathe et al. (2014). 
El algoritmo es aplicable al caso colombiano porque sus criterios poseen un sustento teórico-científico mundial. Si bien la epidemiologia en la FAC puede ser distinta a la analizada en los estudios realizados por los autores, estos no dejan de ser un referente importante. Su adaptación se puede lograr complementando estos estudios con datos de los casos presentados en la población de tripulantes en un periodo de tiempo observable. Con esto, se puede realizar una categorización de eventos de seguridad en términos de factores psicofísicos y de la teoría de los riesgos por patologías en el medio aeronáutico.

En el marco de la investigación Development of an Aeromedical Scientific Information System for Aviation Safety, otros algoritmos fueron desarrollados. Estos algoritmos son el producto de la creación del sistema de información aeromédico. Este sistema es complemento útil a la herramienta, pues permite determinar factores como el estado de los certificados, calcular la fecha de expiración de los certificados, los días efectivos de certificación por tripulante, determinar qué tripulantes han estado involucrados en eventos de seguridad, establecer códigos por patologías y determinar el tipo de certificado a otorgar (Peterman et al., 2008).

\section{Conclusiones}

En el mundo, la certificación aeromédica se ha fortalecido gracias a la evidencia científica. Esta certificación ha pasado de ser un proceso de selección por cumplimiento de requisitos psicofísicos, a ser un proceso de toma de decisiones en el cual confluyen herramientas para disminuir el riesgo por factores psicofísicos en el medio aeronáutico.

El proceso de certificación en la FAC es susceptible de mejora si se incorporan tres procesos que robustecen la ADM, fomentan la investigación en medicina aeroespacial e impactan de manera directa la seguridad aérea. Estos procesos son (1) la creación de un sistema de información aeromédico; (2) la evaluación de matrices de riesgo aeromédico, discriminada por tipos de operación, teniendo en cuenta los cargos y sus requerimientos psicofísicos, y (3) la aplicación de algoritmos como soporte para la ADM.

Implementando una estratificación del riesgo para la certificación se logra otro impacto a nivel operacional, ya que es posible reducir el número de personal con certificación aeromédica (por cuanto los requisitos para determinados cargos de bajo riesgo son menores), sin afectar la seguridad operacional. Los tres procesos propuestos son complementarios entre sí, pero pueden ser desarrollados e implementados de manera independiente. Con base en la evaluación del riesgo, y dependiendo del tipo de certificación requerida, se debe establecer un diferencial de exámenes médicos, lo cual supone una disminución en los costos de los controles psicofísicos actuales.

\section{Referencias bibliográficas}

Department of the Air Force. (2013). AFI 48-123 Medical Examination Standards. Department of the Air Force.

Drane, A. M., Navathe, P., \& Clem, P. (2013) Aeromedical Certification of Aircrew and Controllers with Renal Calculi. Aviation Space Environment Medicine, 84(10), 1074-1081. https://doi.org/10.3357/ASEM.3604.2013

Evans, A. D. B. (2016). Aeromedical Risk a Numerical Approach, Practical Decision Making in aeromedical certification. En D. Gradwell y D. Rainford (eds.), Ernsting's Aviation and Space Medicine ( $5^{\text {th }} \mathrm{Ed}$ ) (pp. 373-382). CRC Press.

Fuerza Aérea Colombiana. (2011). Manual FAC 3-007 público de gestión en seguridad operacional FAC. Fuerza Aérea Colombiana.

Fuerza Aérea Colombiana. (2016). Reglamento de aptitud psicofísica de ingreso y especial para la Fuerza Aérea Colombiana (4ta ed.). Fuerza Aérea Colombiana.

Gray, G., Bron, D., Davenport, E. D., d’Arcy, J., Guettler, N., Manen, O., Syburra, T., Rienks, R., \& Nicol, E. D. (2019). Assessing aeromedical risk: a three-dimensional risk matrix approach. Heart, 105, 9-16. https://doi.org/10.1136 /heartjnl-2018-313052

Ministerio de Defensa Nacional. (2000, 14 de septiembre). Decreto 1796 del 2000. Diario Oficial 44.161. http://www.se cretariasenado.gov.co/senado/basedoc/decreto_1796 _2000.html

Navathe, P., Drane, M., \& Preitner, C. (2014). Aeromedical Decision Making: From Principles to Practice. Aviation 
Space Environment Medicine, 85(5), 576-580. https://doi. org/10.3357/ASEM.3561.2014

Peterman, C. L., Rogers, P. B., Véronneau, S. J. H., \& Whinnery, J. E. (2008). Development of an Aeromedical Scientific Information System for Aviation Safety. Civil Aerospace Medical Institute Federal Aviation Administration.

RAC 67. (2017). Normas para el otorgamiento del certificado médico aeronáutico. Unidad Administrativa Especial de Aeronáutica Civil.

Ricaurte, E. M., Mills, W. D., De John, C. A., Laverde-Lopez, M. C., \& Porras-Sanchez, D. F. (2016). Aeromedical Hazard Comparison of FAA Medically Certified Third-Class and Medically Uncertified Pilots. Aerospace Medicine Hum perform, 87(7), 618-621. https://doi.org/10.3357/ AMHP.4360.2016

United States Department of Defense. (2018, 6 de mayo). DOD Instruction 6130.03. Medical standards for appointment, enlistment, or Induction into the military services. United States Department of Defense. 